\title{
High-throughput Sequencing Study of the Effect of Transabdominal Hysterectomy on Intestinal Flora in Patients with Uterine Fibroids
}

\section{CURRENT STATUS: UNDER REVIEW}

BMC Microbiology $\triangle$ BMC series

Wantong Wang

Shengjing Hospital of China Medical University

Yibing Li

Shengjing Hospital of China Medical University

Qijun Wu

Shengjing Hospital of China Medical University

Xin Pan

Shengjing Hospital of China Medical University

Xinhui He

Shengjing Hospital of China Medical University

xiaoxin ma

Shengjing Hospital of China Medical University

च maxiaoxin666@aliyun.comCorresponding Author

ORCiD: https://orcid.org/0000-0003-0271-8035

DOI:

$10.21203 / \mathrm{rs} .2 .18237 / \mathrm{v} 1$

\section{SUBJECT AREAS}

Applied \& Industrial Microbiology General Microbiology

\section{KEYWORDS}

intestinal flora, uterine fibroids, hysterectomy, estrogen, high-throughput sequencing, 16sRNA 


\section{Abstract}

Background: To investigate the effect of transabdominal hysterectomy on the diversity

of the intestinal flora in patients with uterine fibroids. Patients with uterine fibroids were selected from September 2018 to December 2018, in the Department of Obstetrics and Gynecology, Shengjing Hospital, China Medical University, and stool specimens were collected from patients before and after surgery.

Results: High-throughput sequencing of the 16S rRNA gene was used to detect the changes in microbial community structure and diversity, and the effects of total hysterectomy on the intestinal flora were further analyzed. Estrogen levels decreased after trans-abdominal hysterectomy. Highthroughput sequencing showed that after abdominal hysterectomy, the abundance and diversity of the intestinal flora decreased. The abundance changes were mainly due to Proteobacteria, where their abundance increased.

Conclusions: Trans-abdominal hysterectomy changes the intestinal flora of the body by lowering the level of estrogen in the body, which reduces the diversity and abundance of the intestinal flora.

\section{Full-text}

Due to technical limitations, full-text HTML conversion of this manuscript could not be completed.

However, the manuscript can be downloaded and accessed as a PDF.

Tables

\begin{tabular}{|c|c|c|c|c|}
\hline & \multicolumn{2}{|c|}{ Group } & \multirow[t]{2}{*}{ T value } & \multirow[t]{2}{*}{ P value } \\
\hline & $\operatorname{RS1}(n=15)$ & $\operatorname{RS} 2(n=15)$ & & \\
\hline AMHnmmol/L & $4.78 \pm 1.75$ & $4.01 \pm 1.00$ & 1.82 & 0.09 \\
\hline $\mathrm{E}_{2}(\mathrm{pmol} / \mathrm{L})$ & $460.71 \pm 303.08$ & $229.36 \pm 110.19$ & 2.65 & 0.02 \\
\hline $\mathrm{FSH}(\mathrm{IU} / \mathrm{L})$ & $11.86 \pm 11.77$ & $19.47 \pm 28.29$ & -0.94 & 0.36 \\
\hline
\end{tabular}

Table 1 Comparison of ovarian function between the two groups

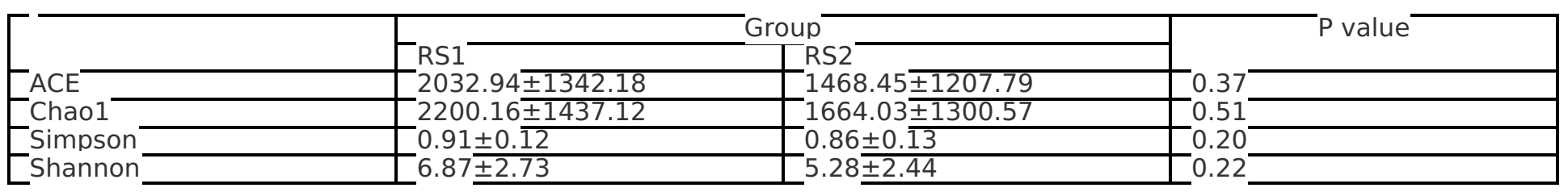

\section{Table 2 Sequencing results and diversity index of two groups of samples}

*The operational taxonomic units (OTUs) were defined at the $97 \%$ similarity level

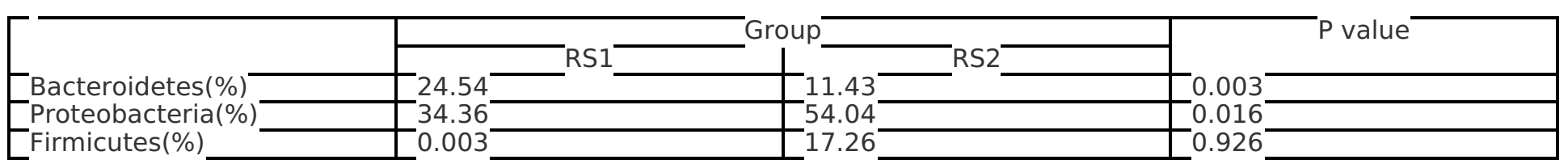


Table 3. Comparison of relative abundance of two groups of TOP3 strains at the phylum level

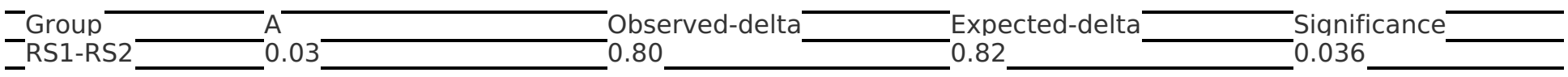

Table 4 Significantness test table for community structure differences between groups

Figures

A

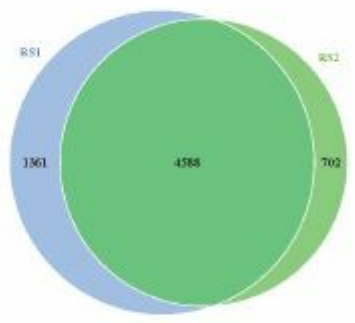

$\mathrm{C}$

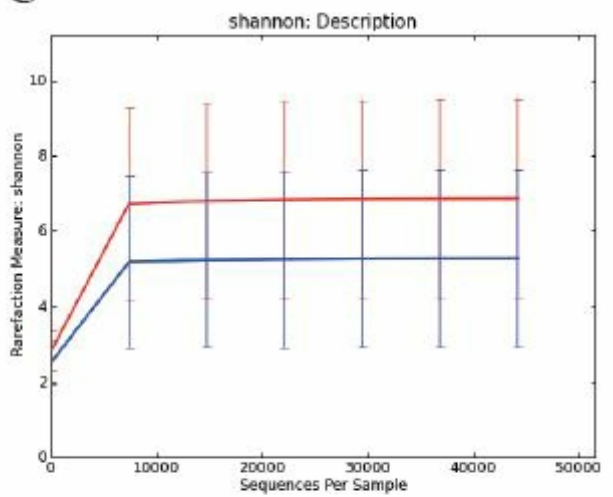

$\mathrm{E}$

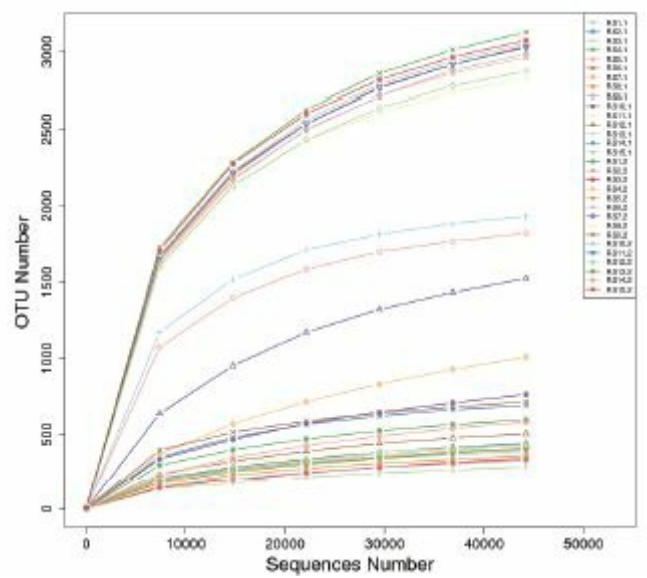

B
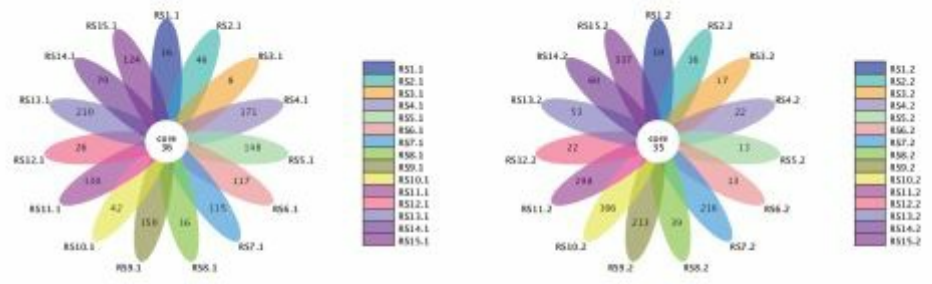

$\mathrm{D}$

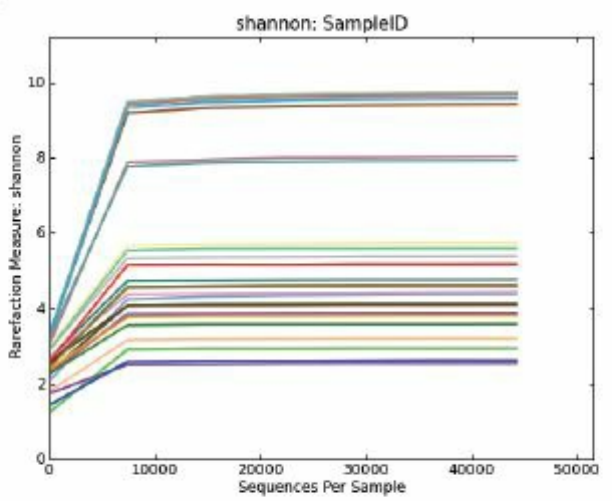

F

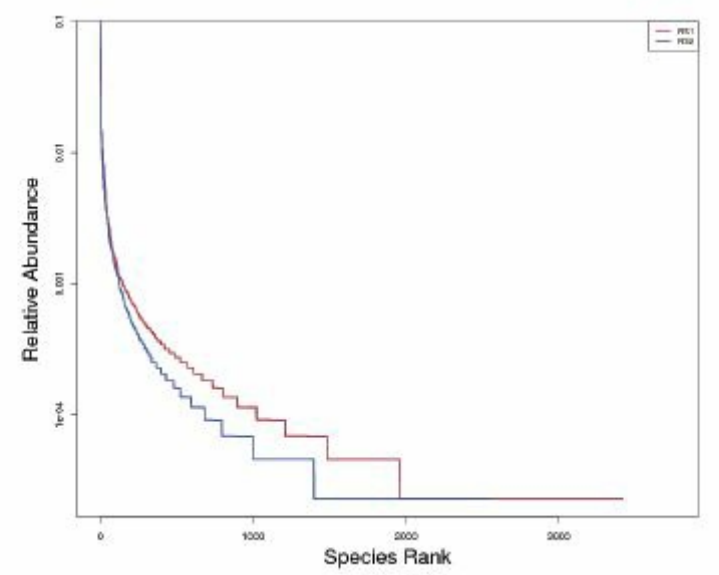

Figure 1 
A
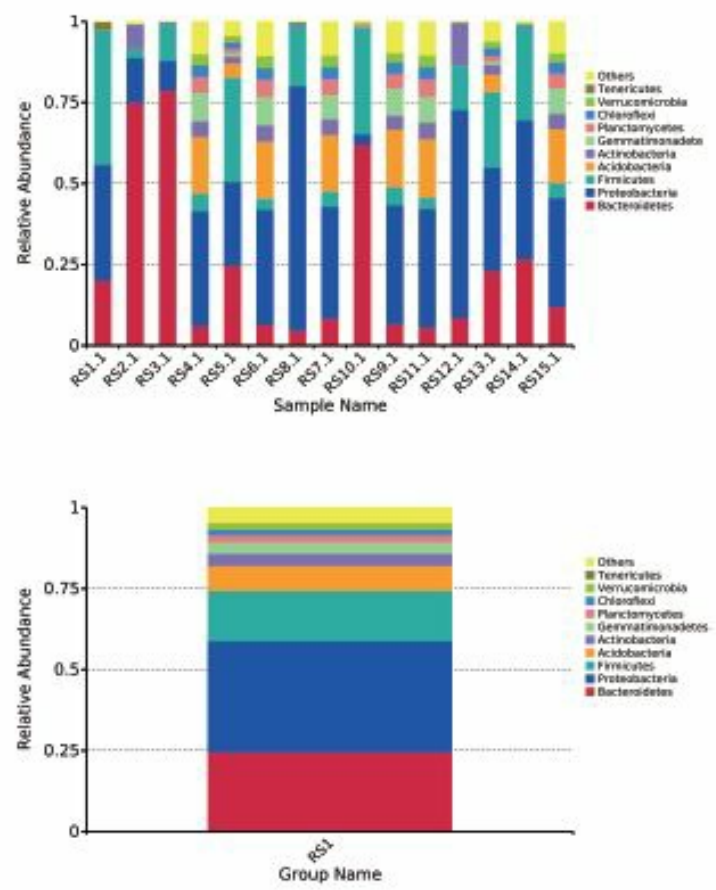

C
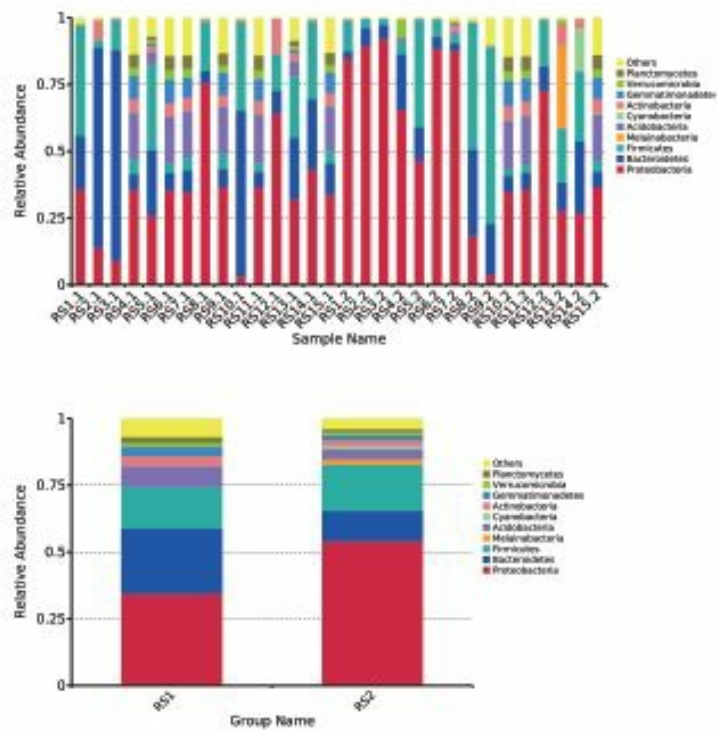

B
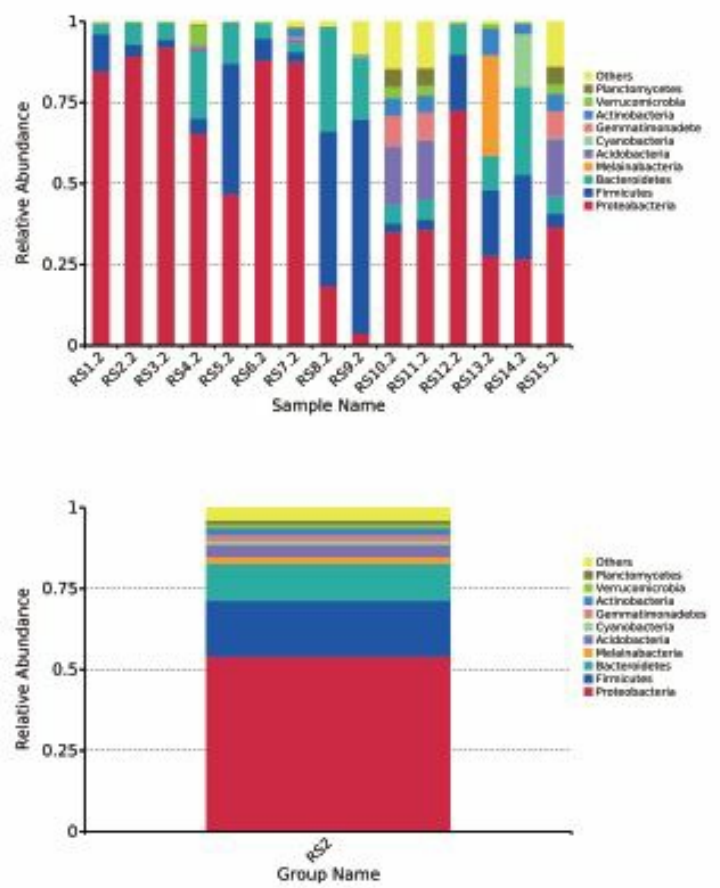

D

$-\infty 61$

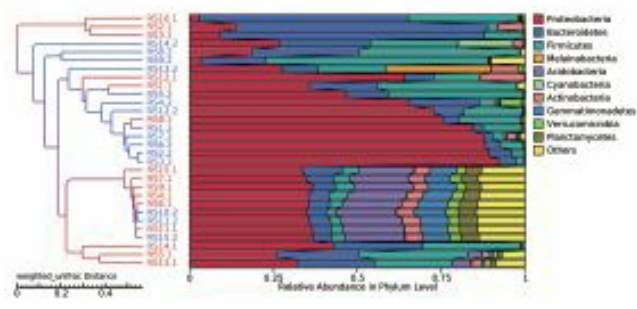

${ }_{-\infty \pi} \mathrm{E}$

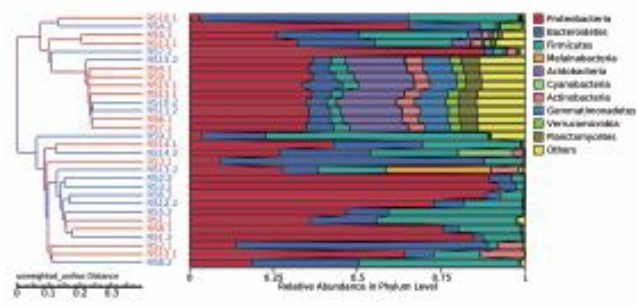

Figure 2 
A
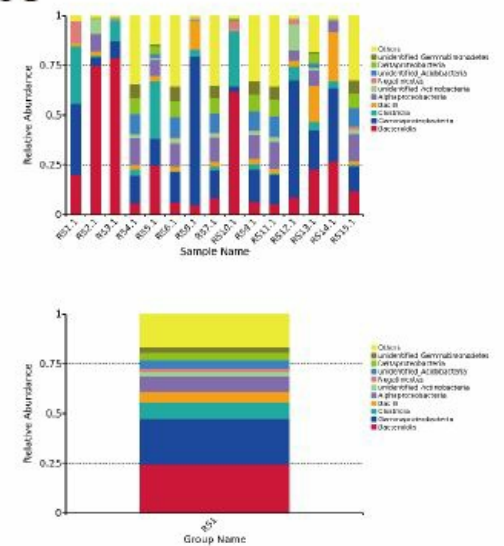

D
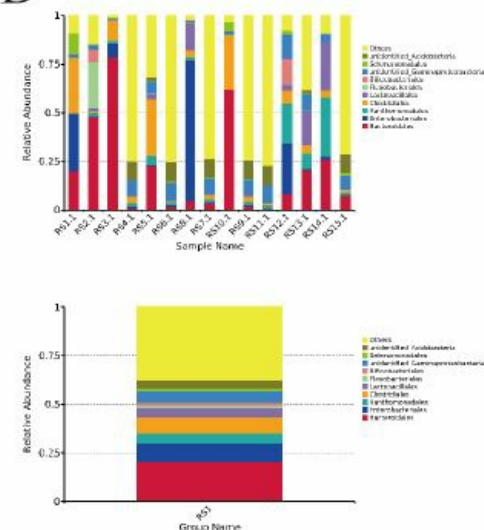

B
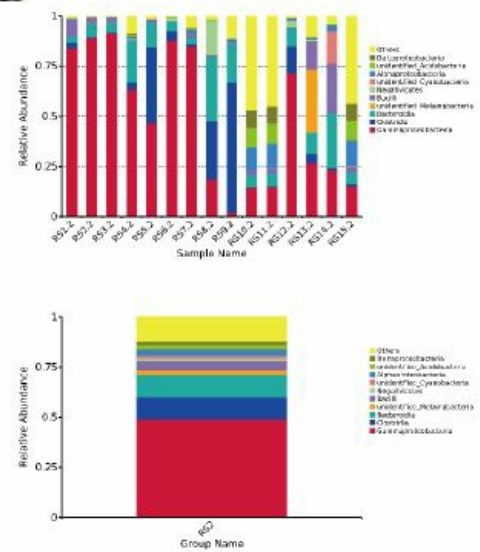

E
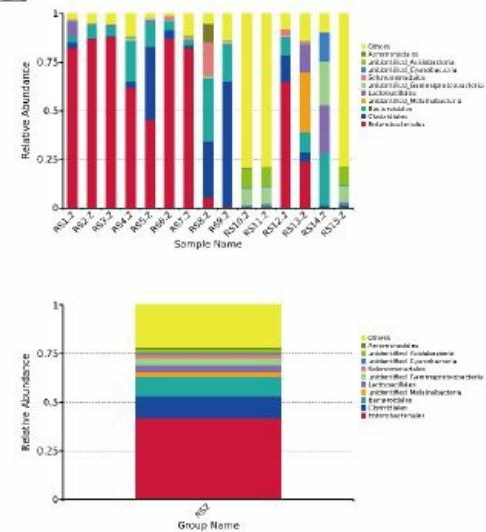

C
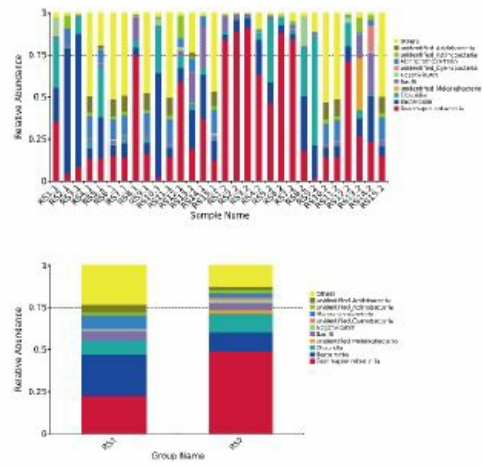

F
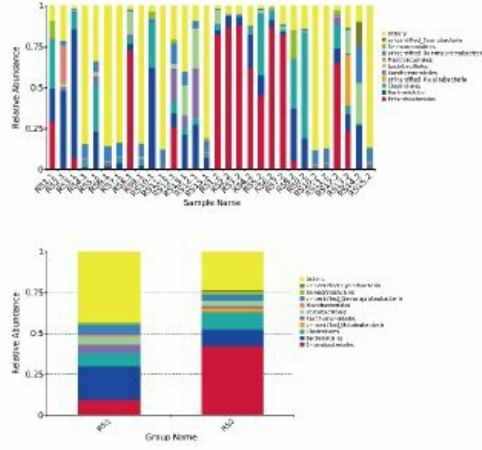

Figure 3 


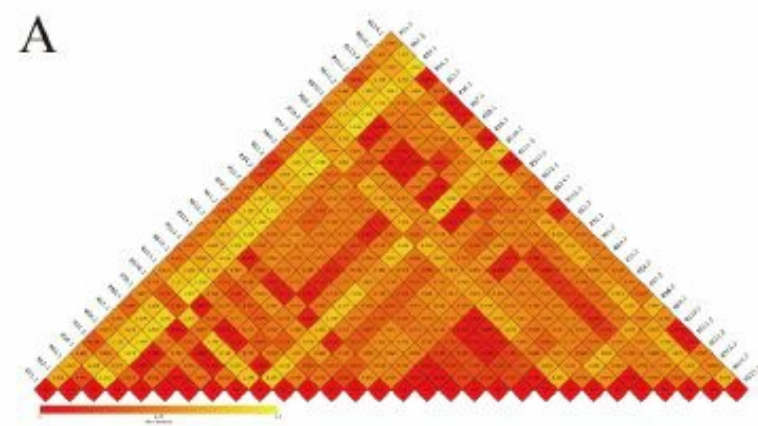

B

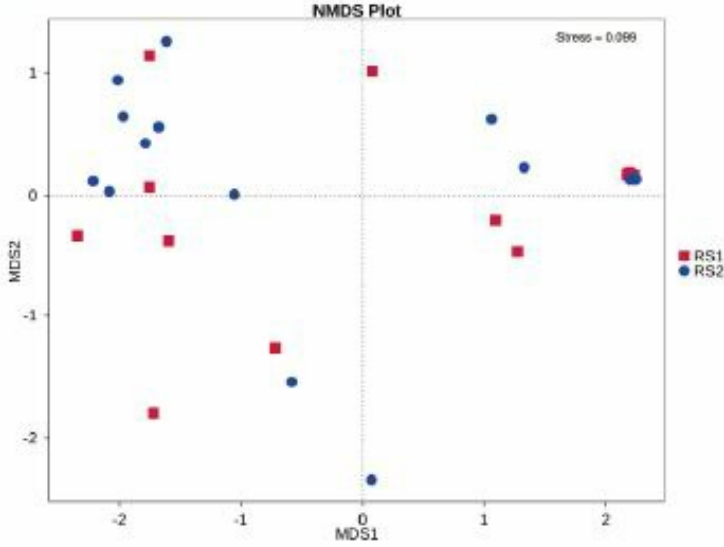

F

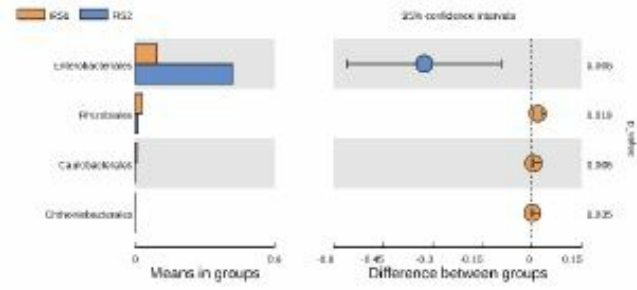

$\mathrm{H}$

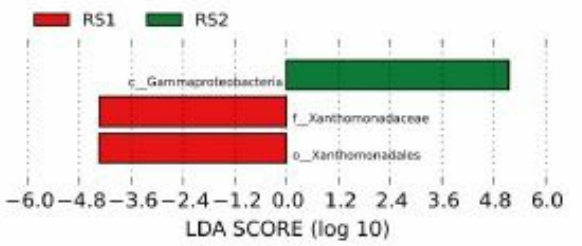

C

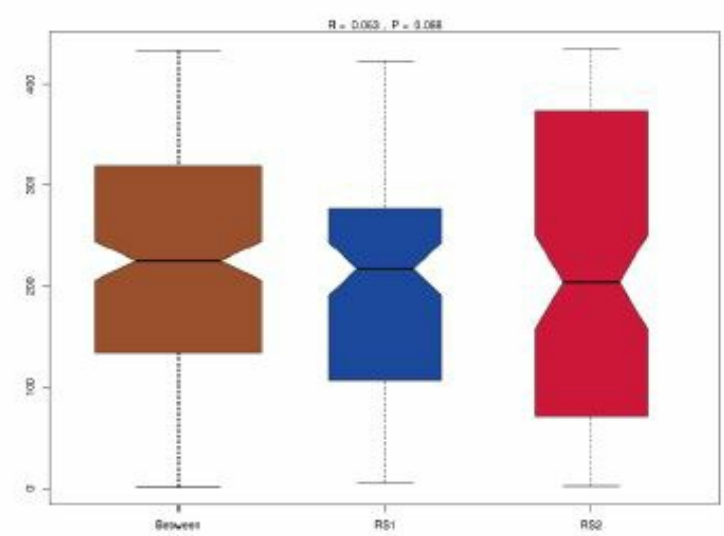

D

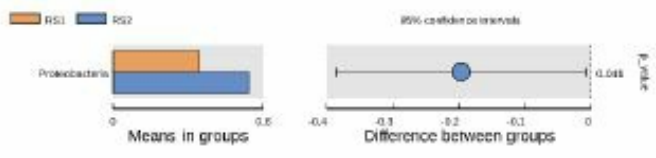

$\mathrm{E}=\ldots$

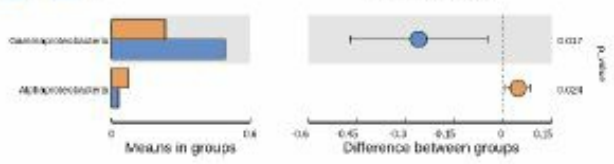

G
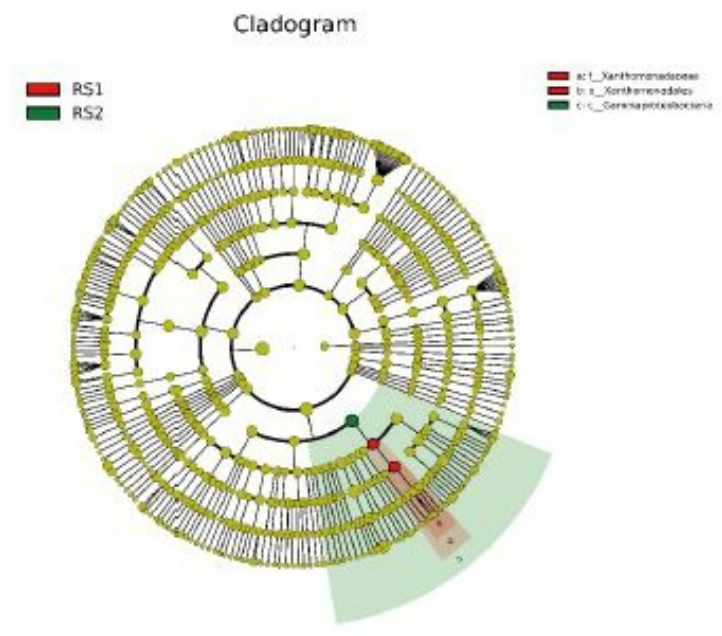

Figure 4 